\title{
Panel de expertos del sector eléctrico: lecciones para el diseño e implementación de políticas públicas en otras áreas reguladas
}

Panel of experts from the electricity sector: lessons for the design and implementation of public policies in other regulated areas

\author{
José Ávila \\ Universidad de Chile
}

\section{Introducción}

El diseño e implementación de políticas públicas de calidad exige un cuidadoso estudio y definición de sus aspectos institucionales ya que "...las orientaciones analíticas mejor fundadas pueden fracasar o no consolidarse en un medio institucional insuficiente o adverso" (Lahera, 2002: 38).

En este contexto, los estudios de benchmarking o de mejores prácticas pueden resultar útiles para identificar experiencias exitosas que sirvan de referente para seleccionar o diseñar los instrumentos institucionales más adecuados para la realización de una determinada política (Lahera, 2002). Para estos efectos, entendemos benchmarking como: "...un proceso sistemático de medición y evaluación de productos, servicios, y prácticas de líderes reconocidos en el terreno, para determinar cuáles pueden ser copiadas o adoptadas para obtener un desempeño superior" (Pollitt, Cave \& Joss, 1994 citado en Lahera, 2002).

En Chile, una experiencia institucional exitosa la constituye el Panel de Expertos creado en la reforma del sector eléctrico del año 2004, como mecanismo especial para la solución de determinados conflictos producidos a propósito de la regulación del sector.

En la institucionalidad de los mercados creados en los procesos de privatización durante los años ?80, se incorporaron progresivamente mecanismos especiales para la solución de los conflictos, por razones de eficiencia económica, ya que estos mecanismos permiten proponer respuestas más adecuadas a la naturaleza técnica del conflicto; otorgan soluciones más expeditas; y permiten controlar la discrecionalidad del Estado dando garantías y certezas a la inversión privada.

El Panel de Expertos ha tenido éxito en reducir el número de conflictos producidos en relación a estas materias y los tiempos de tramitación de las con-

\footnotetext{
*Dirección de correspondencia [Correspondence address]: José Ávila, Universidad de Chile E-mail: josegustavoavila@gmail.com
}

troversias (Fischer et al., 2009); y se constituyó en un elemento esencial de la regulación de los sectores de infraestructura en Chile: eléctrico, telecomunicaciones, servicios sanitarios y concesiones (Jadresic, 2007).

La incorporación de paneles de expertos en la regulación de los sectores indicados, presenta algunas características que la definen. En primer lugar, su incorporación se realiza a través de instituciones propias, desarrolladas a partir de la legislación comercial internacional para los Dispute Boards, pero adaptadas a las necesidades del rubro. Además, se incorporan en mercados regulados, con muy pocos actores que tienen gran poder de mercado, y con una intervención del Estado justificada en la promoción de la eficiencia de los mismos.

Esta intervención se produce habitualmente en dos ocasiones: al momento de definir las bases de los estudios utilizados para el cálculo de tarifas y al momento de efectuar la fijación de las mismas. Una situación especial se produce respecto del Panel de Expertos del Sector Eléctrico donde gran parte de su labor ha consistido en solucionar los conflictos producidos entre las empresas que participan de un mismo CDEC. En estos casos, el Panel ha debido realizar un trabajo de tipo normativo, ya que ha debido determinar cuáles son las normas aplicables a la situación particular y cuál es la correcta interpretación que hay que dar a las mismas.

Por otra parte, los paneles de expertos incorporados en los sistemas regulatorios chilenos no sólo presentan diferencias con los paneles de expertos establecidos en la legislación comercial internacional, sino que además, estos paneles son muy diferentes entre sí, en cuánto a composición, atribuciones y procedimientos.

De esta manera el objetivo general del presente estudio es identificar las mejores prácticas presentes en el diseño institucional del Panel de Expertos que puedan ser utilizadas como referente en el diseño de mejores mecanismos para la solución de conflictos en otras materias. 


\section{Metodología}

En atención al objetivo propuesto, efectuamos un estudio de tipo cualitativo, descriptivo e inductivo, basado en la utilización y análisis de fuentes secundarias como artículos y publicaciones tanto de medios especializados, como de organismos públicos involucrados, leyes y reglamentos, historia de la tramitación de los proyectos de ley, jurisprudencia judicial y administrativa y dictámenes del Panel de Expertos; y en la realización de entrevistas semi estructuradas a stakeholders del sector público, de las empresas reguladas y del mundo académico, consultando su opinión sobre el desempeño del Panel y sobre los aspectos que han influido en el mismo.

Para seleccionar a los entrevistados se elaboró, en primer término, un Mapa de Stakeholders en base a la información recopilada y aplicando la metodología propuesta por Mario Waissbluth y José Inostroza en su Guía Metodológica para el Análisis de Organizaciones (2008).

A partir del mapa elaborado, se realizaron entrevistas a representantes de los siguientes grupos de stakeholders identificados: directivos de las principales empresas del sector, a directivos de pequeñas empresas, a directivos de asociaciones gremiales que agrupan a empresas del sector, a directivos de organismos públicos que interactúan con el panel, y expertos en la materia.

\section{El panel de expertos en el sector eléctrico}

Antes de la creación del Panel, coexistían diferentes formas de resolver conflictos en el sector. Dependiendo del tipo de conflicto, estos debían ser resueltos por distintas entidades administrativas como la Comisión Nacional de Energía, una Comisión Pericial compuesta por ingenieros, una comisión de Hombres Buenos designada por el Ministerio de Energía, o por el Ministro de Energía. Además, determinadas controversias podían terminar siendo resueltas por la Contraloría General de la República al conocer y resolver los reclamos de ilegalidad presentados para impugnar determinados actos de los órganos y autoridades públicas.

La regulación del sector contemplaba también, que otras controversias fueran resueltas por instancias jurisdiccionales como un Tribunal Arbitral conformado especialmente para el caso en cuestión o ante la Corte de Apelaciones respectiva, conociendo de acciones especiales o de acciones jurisdiccio- nales ordinarias, particularmente el recurso de protección.

Este sistema fue objeto de múltiples críticas. Las principales observaciones recogidas apuntan a asimetrías en la información con que cuenta el regulador con respecto a la que poseen las empresas reguladas (Galetovic y Sanhueza, 2002). Además, se critica que tanto el tribunal arbitral como las comisiones periciales se constituyeran para resolver un único conflicto, lo que impedía que existiera aprendizaje y acumulación de conocimiento por parte de los peritos. Además, en los hechos, las partes podían desconocer sus dictámenes y seguir negociando sus posiciones, lo que le restaba valor a la decisión de la Comisión y hacía poco atractivo acudir a la misma, ya que su decisión no resolvía el conflicto.

Otro elemento de crítica tenía que ver con las atribuciones del ejecutivo en la resolución de los conflictos. Vergara Blanco (2005), critica el hecho de que sea un órgano administrativo el que resuelva controversias entre privados, lo que es ajeno a su naturaleza y sus funciones. Además, en el Sector se criticó que este mecanismo se prestaba para mucho lobby político, ya que existía un proceso paralelo e informal que era a través del cual se resolvían, en los hechos, los conflictos.

Otros autores critican el rol de la Contraloría General de la República; Jadresic et al. (2001), sostienen que es un error que este órgano se hubiera constituido en una instancia de apelación, ya que asumía un rol regulador que no le corresponde y para el cual no estaba preparado.

Respecto de las decisiones judiciales sobre los conflictos del sector, Jadresic et al. (2001), señalan que estas presentaban falencias tales como mala calidad técnica, la que se explica en las complejidades técnico económicas de las materias en conflicto y la falta de tiempo para efectuar un adecuado estudio de los mismos; la incertidumbre producida por la impredictibilidad del resultado final del conflicto, producto de esta misma mala calidad; y los largos tiempos necesarios para obtener un pronunciamiento final.

En este contexto, la creación del Panel de Expertos en la reforma del Sector Eléctrico del año 2004 debe ser entendida como una respuesta de la autoridad frente a las demandas del sector, las que se entendieron como legítimas y justificadas.

A principios de 2002, el Ejecutivo presentó un proyecto de ley que pretendía efectuar una serie de reformas en la regulación del sector eléctrico con el objetivo declarado de superar las falencias existentes en la misma y que dificultaban la operación del sector. Tras un proceso de discusión y recibiendo las 
críticas y sugerencias formuladas por los académicos y representantes del Sector, se incluyó en el proyecto la creación de un panel de expertos, para conocer y decidir respecto de determinadas controversias entre el regulador y las empresas. Finalmente, el proyecto fue aprobado en marzo de 2004. En el artículo $3^{0}$ de dicha ley se agregó un nuevo Título VI a la Ley General de Servicios Eléctricos en el que se reguló la organización y funcionamiento del $\mathrm{Pa}$ nel de Expertos. La designación de los integrantes del primer Panel se efectuó el 15 de julio de 2004, por Resolución Exenta № 43 de mismo Ministerio.

En el diseño de este Panel se incluyeron elementos destinados a superar las falencias detectadas en los mecanismos preexistentes. Así se le dio al Panel un carácter permanente y se definió que estuviera conformado por siete miembros, de los cuales cinco deben ser ingenieros o licenciados en ciencias económicas y dos abogados. Además, se estableció que los integrantes del Panel debían ser profesionales de amplia trayectoria profesional o académica y con experiencia mínima de tres años en materias técnicas, económicas o jurídicas del sector eléctrico, los cuales deben ser designados por el Tribunal de Defensa de la Libre Competencia a través de concurso público, por un período de seis años, debiendo renovarse - parcialmente- por mitades, cada tres años. Además se establecen criterios para decretar incompatibilidades e inhabilidades de parte de sus integrantes ante determinadas circunstancias.

A este órgano eminentemente técnico se le entregó el conocimiento y la solución de los conflictos que antes le correspondía al Tribunal Arbitral, a la Comisión Pericial y al Ministro de Economía, a los que se le agregaron otras materias relacionadas con la fijación de tarifas y determinación de valores. A objeto de asegurar una pronta solución al conflicto, se establecieron breves plazos para que el Panel conociera y resolviera la controversia.

El otro recurso utilizado para obtener una rápida solución a los conflictos fue otorgar, a la decisión del Panel, el carácter de definitiva y final. Para ello, la ley estableció que el dictamen o pronunciamiento del Panel es obligatorio para todos los que participen en el procedimiento respectivo y definitivo, ya que en contra del mismo no procede ninguna clase de recursos, jurisdiccionales o administrativos, de naturaleza ordinaria o extraordinaria. Aunque, respecto de algunas materias, la ley estableció que el ministro presidente de la Comisión Nacional de Energía, con acuerdo del Consejo Directivo, puede suspender sus efectos. Este mecanismo no ha sido utilizado, con lo que el carácter definitivo de la decisión del Panel se ha mantenido y reforzado.

Hasta diciembre de 2011, fecha de corte para el presente estudio, se habían presentado ante el $\mathrm{Pa}$ nel 136 discrepancias. De ellas, 3 fueron declaradas inadmisibles y las otras 133 resueltas por dictamen del Panel. El mayor porcentaje de discrepancias $(35,3 \%)$, tratan de conflictos producidos entre empresas al interior de un CDEC; en segundo término, a la fijación del Valor Nuevo de Reemplazo $(28,7 \%)$; en tercer lugar, a la fijación del peaje de subtransmisión $(10,3 \%)$ y en un segundo nivel, con porcentajes menores a $10 \%$ pero mayores de $5 \%$, la determinación de los costos de explotación para las empresas distribuidoras $(8,8 \%)$ y la fijación de precios de los servicios no consistentes en el suministro de energía $(5,1 \%)$. En síntesis, la gran mayoría de las discrepancias $(88,2 \%)$, se origina en las cinco materias mencionadas.

Ahora bien, revisando el contenido específico de estas discrepancias, podemos identificar dos grandes categorías de conflictos que le ha tocado conocer al Panel: discrepancias de las empresas con el regulador y discrepancias entre empresas.

La mayoría de las discrepancias conocidas por el Panel $(63,2 \%)$, corresponde a diferencias producidas entre empresas y organismos reguladores a propósito de las decisiones tomadas por estos últimos y que dicen relación con la determinación de valores, precios y tarifas. Otra parte importante de las discrepancias conocidas por el Panel $(36,8 \%)$, corresponde a diferencias producidas entre las empresas al interior del CDEC, y que dicen relación con la forma de entender y aplicar las distintas normas que regulan la operación de los mismos. En estos casos, lo que se solicita al Panel es que determine si las decisiones adoptadas por estos órganos internos de los CDEC se ajustan a la regulación establecida para una determinada materia y que, de no ser así, establezca cuales son las normas pertinentes y corrija la decisión adoptada. Así, para solucionar este último grupo de controversias, el Panel debe efectuar un trabajo de tipo normativo ya que debe determinar cuáles son las normas que se aplican al caso y cuál es el alcance de las mismas.

De esta forma, y a diferencia de otras instituciones similares, el Panel de Expertos ha desempeñado una doble función: como instancia de reclamación por determinadas decisiones adoptadas por los organismos regulatorios y como instancia para solucionar los conflictos producidos entre las empresas que participan de un mismo CDEC a propósito de la interpretación de las normas que regulan su funcionamiento. 


\section{Efectos de la incorporación del panel}

Consultados sobre los efectos de la incorporación del Panel de Expertos a la institucionalidad del Sector Eléctrico, la mayoría de los entrevistados (14 de 15), consideró que esta incorporación había sido muy positiva ya que había provocado una notoria mejora respecto de la situación preexistente. Solo uno de los entrevistados señaló que consideraba dicha incorporación como positiva, pero con reparos, ya que, siendo una buena idea, se habían cometido una serie de errores en su implementación, lo que hace que las decisiones del Panel no sean del todo adecuadas.

Consultados sobre los aspectos de la solución de conflictos que habían mejorado con la creación del Panel, la mejora reconocida por el mayor número de entrevistados (11), mencionó la disminución de los tiempos necesarios para solucionar los conflictos. El segundo aspecto más mencionado por los entrevistados (6 menciones), es la calidad técnica de las decisiones del Panel, la que atribuyen a la formación técnica y profesional y el conocimiento que del sector eléctrico poseen las personas encargadas de resolver los conflictos.

Otro aspecto mencionado por los entrevistados como un avance es la generación de criterios de decisión consistentes ( 5 menciones). Dos de ellos hablaron expresamente de la existencia de una suerte de "jurisprudencia" del Panel que permitía, en algún grado, anticipar el resultado de una determinada discrepancia. El efecto de este avance es hacer más predecibles las decisiones del Panel y, por lo mismo, otorgar mayor certeza al mercado.

Otro de los efectos positivos provocados por la incorporación del Panel, fue la disminución en la conflictividad del Sector (5 menciones) debido a que provoca un efecto disuasivo sobre los actores del Sector antes de generar una divergencia. Por lo mismo, las empresas han preferido negociar directamente y llegar a acuerdos en vez de acudir al Panel.

Además se mencionan como mejoras, la disminución en los costos financieros (La incorporación del Panel habría equiparado las condiciones para todas las empresas del sector, al otorgar igual derecho a reclamar, y en las mismas condiciones, con independencia de sus capacidades financieras), y el carácter definitivo de las resoluciones.. Por último, algunos de los entrevistados consideraron que otro avance importante, respecto de la situación pre existente, fue la separación de la función reguladora de la función de solución de las controversias. Con ello se impide que la autoridad ocupe la regulación para resolver conflictos particulares y, por otro lado, que se utilice la situación de conflictos específicos para regular, como ocurría en el pasado.

Factores que han incidido en el buen desempeño del panel Se pidió a los entrevistados que señalaran cuáles eran, en su opinión, los factores o elementos que han permitido que el Panel pueda dar una adecuada solución a las controversias que le toca resolver. Las menciones efectuadas por los entrevistados se agrupan en cuatro grandes categorías: factores relacionados con el diseño institucional del Panel; factores relacionados con la forma en que se implementó en los hechos el Panel; factores relacionados con el desempeño del Panel; y, factores relacionados con las características particulares que presenta el sector eléctrico.

Cabe destacar que la mayor cantidad de menciones efectuadas por los entrevistados (52 sobre un total de 69), se refirieron a factores relacionados con el diseño institucional del Panel.

Sobre este aspecto, nueve de los entrevistados señalan que el carácter técnico del Panel es uno de los factores que más incide en el buen desempeño del mismo, ya que los conflictos que le corresponde conocer al Panel son de gran complejidad técnica.

Otro factor clave en el buen desempeño del Panel (9 menciones), ha sido el carácter multidisciplinario del mismo, lo que ha permitido que al momento de estudiar y resolver la discrepancia, se puedan comprender más cabalmente los distintos aspectos de la controversia que le toca conocer y, por lo mismo, dar una solución más adecuada a la misma.

La forma en que el Panel debe resolver las discrepancias, el arbitraje de oferta final, es otro factor que ha contribuido a que el Panel pueda dar adecuada solución a los conflictos. Según señalan los entrevistados, el hecho de que el Panel deba escoger entre alguna de las posiciones presentadas por las partes, sin que pueda proponer posiciones distintas o intermedias, produce efectos benéficos respecto de la solución de los conflictos del sector: evita que los discrepantes adopten comportamientos estratégicos y extremen sus posiciones para obtener un mejor resultado; obliga a fundamentar muy bien las posiciones que se presentan al Panel y a ser responsables en el ejercicio del derecho a acudir al Panel, ya que no se puede improvisar. Además, evita que el Panel se transforme en un organismo regulador, ya que su dictamen se debe limitar a escoger alguna de las posiciones presentadas y facilita el trabajo del Panel, ya que el mismo no debe encontrar la solución óptima al conflicto, sino que debe optar por una de las soluciones propuestas. 
Seis de los entrevistados señalaron que la ausencia de recursos administrativos y judiciales era otro de los factores que había contribuido al éxito del Panel. Según se señaló, el hecho de que el dictamen del Panel no sea apelable es la única garantía de que la discrepancia se resuelva de forma rápida y con conocimiento técnico especializado, lo cual se ve reforzado por la existencia de breves plazos para resolver.

Algunos entrevistados mencionaron que la duración de los integrantes en sus cargos (seis años), era un factor que favorece el buen desempeño del Panel pues permite dar continuidad al trabajo del mismo. Por otra parte, la independencia del Panel respecto de los órganos públicos encargados de la regulación del sector y respecto a las partes en conflicto también ha favorecido su buen desempeño.

Otros factores de diseño mencionados son el hecho que sus procedimientos se encuentren regulados de forma detallada; el número de integrantes del Panel, y el que estos sean seleccionados y remunerados de forma adecuada; además del carácter público de los procedimientos y la especificidad de su función.

Un segundo grupo de factores, dice relación que la selección de sus integrantes; ocho de los entrevistados señalaron que la trayectoria profesional ha resultado determinante en el buen desempeño del Panel, debido al profundo conocimiento del mercado eléctrico y de sus particularidades que se requiere para dar una solución adecuada a los mismos; además es necesario que, junto a los panelistas con amplio conocimiento del Sector, existan también panelistas con una amplia formación en economía.

La calidad de los dictámenes elaborados por el Panel (2 menciones), es otro factor que ha contribuido al éxito del mismo, pues la misma permite a los regulados predecir la opinión del Panel ante una determinada controversia. Además, la calidad técnica del dictamen hace más aceptable la decisión del Panel, y el prestigio alcanzado por el Panel favorece su buen desempeño porque contribuye al acatamiento de sus decisiones. Finalmente, uno de los entrevistados mencionó como factor que contribuye al buen desempeño del Panel, que la mayoría de sus decisiones son adoptadas por unanimidad.

Por último, encontramos factores relacionados a características propias del sector eléctrico. Dos de los entrevistados señalaron que uno de los factores que ha favorecido el desempeño del Panel era la existencia de una cultura de coordinación entre los distintos agentes del mercado eléctrico. La existencia de esta "tradición" permite entender el particular rol dado al Panel como solucionador de conflictos entre empresas.
Otro factor que ha contribuido al buen desempeño del Panel (1 mención), es el aprendizaje que han realizado las empresas y los organismos públicos, a partir de los resultados obtenidos en las discrepancias presentadas ante el mismo, ya que la solución de conflictos por arbitraje de oferta final, ha obligado a empresas y organismos públicos a efectuar mejores investigaciones para poder efectuar presentaciones mejor fundadas. Así, la presencia del Panel, ha provocado una suerte de retroalimentación en el sector, obligando a todos los agentes a realizar un trabajo más exhaustivo al momento de preparar sus presentaciones.

\section{Factibilidad de incorporar el panel de expertos como meca- nismo de solución de conflictos en otros sectores regulados}

La mayoría de los entrevistados (9 menciones), señaló que aun cuando es recomendable, depende de las características del sector regulado y del tipo de controversias que se producen al interior del mismo, pues el Panel de Expertos no es un mecanismo apto para resolver cualquier clase de conflictos. Otros entrevistados (4 menciones), recomendarían la incorporación de mecanismos similares al Panel de Expertos para la solución de conflictos en otros sectores regulados, sin señalar condiciones, mientras que algunos (2), prefirieron no opinar sobre este punto ya que la existencia del Panel de Expertos se justifica por las particularidades del sector eléctrico e ignoraban si estas se presentan en otros sectores.

Consultados sobre las condiciones que deberían presentarse en un determinado sector para que mecanismos como el Panel de Expertos contribuyera a una mejor solución de sus conflictos, los entrevistados (5 menciones), señalaron que la existencia de un Panel de Expertos se justifica en sectores donde existen conflictos asociados a los procesos de fijación de tarifas.

Otra condición que debe darse para que se justifique la creación de un órgano como el Panel de Expertos (3 menciones), es la existencia en el sector respectivo de múltiples agentes que necesiten coordinarse para operar y en donde existan conflictos de alta complejidad técnica, económica y legal que puedan ser resueltos por arbitraje de oferta final.

Otro de los requisitos que deben darse para que la incorporación del Panel de Expertos sea factible (1 mención), es la existencia de un conjunto de princi- 
pios técnicos y económicos ampliamente compartidos por los distintos agentes del sector. Finalmente, uno de los entrevistados señaló que uno de los requisitos para que la presencia de un Panel de Expertos contribuya a una mejor solución de sus conflictos, es la estabilidad de la industria en el tiempo, ya que si existen continuos cambios tecnológicos, como ocurre en el sector telecomunicaciones, estos impiden que se pueda dar continuidad en los criterios para la solución.

Consultados sobre los casos específicos en que podría ser útil la creación de un órgano similar al Panel de Expertos, los entrevistados mencionaron a telecomunicaciones (4 menciones), empresas sanitarias (3 menciones), transportes (2 menciones) y el mismo sector eléctrico, en otras áreas (2 menciones).

\section{Aspectos del funcionamiento del planel de expertos que podrían ser mejorados}

Finalmente, y como parte de la evaluación del trabajo del Panel, se pidió a los entrevistados que formularan las críticas que pudieran tener y que efectuaran las propuestas de reforma que consideraran pertinentes.

Uno de los problemas del funcionamiento del Panel (3 menciones), es la existencia de situaciones en que se presentan varias discrepancias al mismo tiempo, lo que obliga al Panel a trabajar a paso forzado y contra el tiempo, lo que incide en una menor calidad de los dictámenes. Por otra parte, dos de los entrevistados señalaron como crítica que el Panel favorece a la autoridad cuando esta interviene como parte en alguna discrepancia, quien además utiliza al Panel para resolver temas regulatorios a través de la presentación de discrepancias, lo que desvirtúa el sentido de su trabajo.

Uno de los entrevistados criticó la falta de transparencia que existe en el proceso de selección y nombramiento de los panelistas. Ello, porque las empresas no tienen posibilidad de conocer quiénes son los candidatos antes de su nombramiento, y todos los panelistas son escogidos por una única autoridad

Además, entre los entrevistados se mencionaron críticas al excesivo formalismo que ha ido adquiriendo el trabajo del Panel, a la postergación de los aspectos jurídicos en algunas decisiones, y a una disminución progresiva en la calidad de los análisis y justificación de los dictamines.
Por último, se considera que hay casos más conceptuales como, por ejemplo, las relacionadas con determinación de metodologías o mecanismos de cálculo, en las que las controversias no pueden ser resueltas por arbitraje de oferta final, por lo que el Panel se ve obligado a realizar un trabajo que es más propio de un tribunal, pues debe interpretar normas y definir criterios para lograr una decisión, lo que provoca que las decisiones del Panel sean más vagas $\mathrm{y}$, por lo mismo, que a la autoridad y a las empresas les resulte difícil implementar las decisiones del mismo.

Ante la pregunta por mejoras que se puedan sugerir al Panel, encontramos diversas propuestas. Tres de los entrevistados recomendaron revisar las competencias atribuidas al Panel pues, existen materias que este debe conocer, pero que no pueden ser resueltas adecuadamente por él y, además, existen otras controversias en el sector eléctrico que podrían ser resueltas de mejor manera si esta decisión le correspondiera al Panel.

También se recomienda flexibilizar los plazos establecidos en la ley para que el Panel emita su dictamen porque existen discrepancias que requieren un mayor tiempo de estudio para su adecuada resolución; permitir al Panel obtener asesoría externa en materias específicas que no son propias de su experticia; limitar las actividades académicas de los panelistas y establecer una dedicación mínima a sus cargos; que el Panel funcione en dos salas, a objeto de que cada una de ellas pueda revisar las decisiones de la otra; y establecer la obligación de notificar a los demás integrantes del CDEC antes de presentar una discrepancia al Panel, lo que presenta ventajas pues permite que todas las empresas tengan un plazo razonable para preparar adecuadamente sus presentaciones ante el Panel y les facilita llegar a acuerdos directos, antes que la discrepancia sea presentada al mismo.

\section{Conclusiones}

A partir del análisis de las entrevistas realizadas y de las fuentes secundarias revisadas para el presente estudio, es posible llegar a algunas conclusiones. En primer lugar, el Panel de Expertos fue incorporado como mecanismo especial de solución de determinados conflictos en el sector eléctrico con el propósito de superar una serie de falencias que presentaban las formas pre existentes y que generaban incertidumbre. Los aspectos más relevantes considerados en el diseño institucional del Panel de Expertos persiguen el logro de este objetivo.

El Panel de Expertos ha tenido éxito en mejo- 
rar la solución de conflictos en el sector en varios aspectos: ha reducido notablemente el tiempo necesario para resolver estos; ha avanzado en la calidad técnica de las decisiones; ha permitido mantener los criterios de decisión en el tiempo; ha reducido la conflictividad del sector; ha implicado menores costos financieros; ha permitido separar la solución de conflictos de la regulación y solucionar estos de manera definitiva; y ha homologado las condiciones para los distintos agentes del sector.

El éxito del Panel de Expertos se debe, en gran parte, a determinados aspectos institucionales considerados en su diseño: su carácter técnico y multidisciplinario; la existencia de plazos breves para resolver; su independencia respecto de los órganos públicos y de las empresas; la publicidad del procedimiento; y, especificidad de su función. Estos aspectos institucionales del Panel pueden ser considerados como ejemplos o referentes al momento de diseñar mecanismos de solución de conflictos eficaces y eficientes en otros sectores regulados. A pesar de su buen desempeño, existen ciertos aspectos del diseño institucional del Panel de Expertos que deben ser mejorados.

Aun cuando el Panel de Expertos ha tenido éxito, esta institución no es necesariamente replicable en otros sectores regulados, ya que su buen desempeño se debe, en parte, a la adecuación de la institución a determinadas características del mercado eléctrico y de los conflictos que a su interior se producen. Por ello, la conveniencia de incorporar determinados aspectos institucionales del Panel en los mecanismos de solución de conflictos de otros sectores regulados es relativa, y dependerá de las características de cada sector.

\section{Referencias}

Fischer, R., Palma, R., y Guevara, J. (2009). El Panel de Expertos como Mecanismo de Resolución de Conflictos en el Sector Eléctrico - El Caso de Chile. Serie Economía NAं 260, pp. Documento de trabajo, Centro de Economía Aplicada, Departamento de Ingeniería Industrial, Universidad de Chile.

Galetovic, A. y Sanhueza, R. (2002). Regulación de los Servicios Públicos: Â¿Hacia dónde debemos ir? Revista Estudios Públicos, (85).

Jadresic, A. (2007). Solución de controversias mediante comisiones de expertos en Chile. Serie en Foco, (101).

Jadresic, A., Blanlot, V., y San Martín, G. (2001). La nueva regulación. Dolmen, Santiago de Chile. Lahera, E. (2002). Introducción a las políticas públicas. Fondo de Cultura Económica, Santiago.

Vergara Blanco, A. (2005). El Contencioso Administrativo en Materia Eléctrica: Naturaleza Jurisdiccional de las Funciones del Panel de Expertos. Revista de Derecho de la Universidad Finis Terrae, (9):207-229.

Waissbluth, M. e Inostroza, J. (2008). Guía Metodológica para el Análisis de Organizaciones. Documento de Trabajo. Departamento de Ingeniería Industrial, Universidad de Chile. 